\title{
The Contribution of Semantic Features to the White Matter Pathways of Tool Processing
}

\author{
Jet M. J. Vonk \\ Speech-Language-Hearing Sciences PhD Program, The Graduate Center of the City University of New York, New York, New York 10016 \\ Review of Bi et al.
}

Humans can conceptually organize many objects and actions into semantic categories (e.g., animals, tools). But what is the underlying neurobiology of such a categorical organization? For gray matter, the cortical organization underlying the semantics of both tool objects and tool actions has been extensively studied. A broad network of gray matter structures is thought to be simultaneously activated during tool processing, ranging from bilateral frontal regions [i.e., dorsolateral premotor cortex (DLPMC), ventral premotor cortex (VPMC), and inferior frontal gyrus (IFG)] to bilateral temporal [i.e., fusiform gyrus (FFG) and posterior middle temporal gyrus (pMTG)] and bilateral parietal regions [i.e., inferior parietal lobe (IPL) and superior parietal lobe (SPL)] (Lewis, 2006).

In contrast to gray matter regions, not a lot is known about either the structure or functionality of white matter pathways involved in the semantic processing of categories, including those of tool objects and actions. Identifying which white matter tracts are involved in the semantic network

Received June 26, 2015; revised Aug. 2, 2015; accepted Aug. 5, 2015.

This work was supported by grants from Fulbright Scholarship Program (via Netherland-America Foundation), Prince Bernhard Culture Foundation (via Banning-de Jong Fund), and Alzheimer Nederland. I thank Dr. Loraine Obler, and Katherine Dawson, Eve Higby, Marta Korytkowska, and Amy Vogel for helpful comments and editing.

Correspondence should be addressed to Jet M. J. Vonk, The Graduate Center of the City University of New York, Speech-Language-Hearing Sciences PhD Program, 365 Fifth Avenue, New York, NY 10016. E-mail: jmjvonk@gmail.com.

DOI:10.1523/JNEUROSCI.2453-15.2015

Copyright $\odot 2015$ the authors $\quad 0270-6474 / 15 / 3512609-03 \$ 15.00 / 0$ is crucial, as these tracts connect the involved cortical regions and potentially support the communication between different pieces of semantic information. A recent article in The Journal of Neuroscience by Bi et al. (2015) directly addressed the functional role of white matter connections in the semantic processing of tool concepts (i.e., objects) and tool use (i.e., actions). The authors provide convincing evidence for an underlying structural network necessary for tool processing consisting of intrafrontal, frontoparietal, and frontotemporal tracts.

One important argument in establishing functional specificity within a brain region is that neuroimaging in healthy participants does not distinguish whether a certain region is necessary to access semantic information or if it might be activated because of a secondary, epiphenomenal effect (Mahon and Caramazza, 2008). However, if certain gray matter regions or white matter tracts are essential for semantic processing, then neurological impairment (e.g., caused by stroke, traumatic brain injury, or dementia) in those regions or tracts should result in decreased behavioral performance (Libon et al., 2013). This is a strength of the study by $\mathrm{Bi}$ et al. (2015), which tested whether the integrity of specific white matter tracts was correlated with the behavioral responses of a large patient population. As a result, the authors established three white matter pathways for tool use and eight white matter pathways for tool objects, whose connections are necessary for intact behavioral performance on tool-related tasks. This study, then, is able to contribute an important set of findings to the debate on what neural structures are crucially involved in tool processing. However, it would be interesting to consider how smaller pieces of semantic information (i.e., semantic features such as "contact," "motor-action," and "function") can explain some of their results in more detail, which will be the focus of this short review.

Bi et al. (2015) first identified, in 49 healthy participants, 33 white matter connections among 14 bilateral gray matter regions related to tool processing in previous studies (Lewis, 2006). They subsequently tested the integrity of these white matter tracts in 86 brain-damaged patients via structural imaging (lesion percentage) and diffusion imaging [mean fractional anisotrophy (FA) value]. Patients were tested behaviorally on tool-related action and object tasks and non-tool-related control tasks. In a tool-use task, patients were given 10 common tools (e.g., scissors, broom, iron), and they had to show how these tools were typically used; their responses were evaluated on measures of appropriateness and postural and kinematic accuracy. A non-tool imitation task with intransitive actions (e.g., applaud, salute) served as the control task. Three tool concept tasks included oral picture naming (i.e., speak out the name of a colored photograph), picture-associative matching (i.e., judge which of two colored photographs is semantically closer to a third target image), and word-associative matching (i.e., equal to picture-associative matching, but with words) of common tools, which were also administered with two 
Table 1. Comparison of difference in partial correlations with and without controlling for the other tool task

\begin{tabular}{|c|c|c|c|c|}
\hline & \multicolumn{4}{|c|}{ Partial correlations (original - covariated = difference) } \\
\hline & \multicolumn{2}{|c|}{ Tool use (covariate: tool concept) } & \multicolumn{2}{|c|}{ Tool concept (covariate: tool use) } \\
\hline & Lesion analysis & FA analysis & Lesion analysis & FA analysis \\
\hline Left DLPMC-left IFG & $-0.47--0.38=0.09$ & $0.38-0.32=0.06$ & $-0.43--0.28=0.15$ & $0.38-0.23=0.15$ \\
\hline Left DLPMC-left VPMC & $-0.46--0.39=0.07$ & $0.39-0.34=0.05$ & $-0.40--0.24=0.16$ & $0.35-0.20=0.15$ \\
\hline Left IFG-left IPL & $-0.41--0.28=0.13$ & $0.32-0.21=0.11$ & $-0.47--0.35=0.12$ & $0.45-0.35=0.10$ \\
\hline
\end{tabular}

Based on data from Tables 3 and 4 in Bi et al. (2015).

non-tool categories, namely "animals" and "people."

White matter tract integrity (both lesion percentages and mean FA values) was correlated with the tool-use appropriateness score on three white matter tracts: left IFGleft IPL, left DLPMC-left IFG, and left DLPMC-left VPMC. Moreover, less integrity of the left IFG-left IPL (a frontoparietal tract) was associated with lower kinematic scores, while less integrity of the left DLPMC-left IFG and left DLPMC-left VPMC (two intrafrontal tracts) was associated with lower postural scores. Previous research has suggested that the gray matter regions connected by these tracts are associated with motor planning, motor control, object manipulation, and action production and understanding (for review, see Bi et al., 2015). Additional analyses on tool use with the tool-concept composite score and the intransitive action control task as covariates showed that these tracts uniquely contribute to processing tool use. For tool objects, tract integrity correlated with a composite score of the three tool conceptual tasks on eight white matter tracts: the same three tracts as for tool use in addition to the left IFG-left SPL, left IPL-left VPMC, left SPL-left VPMC, left IFG-left pMTG, and left pMTG-left VPMC (Bi et al., 2015, their Table 3). However, when "animals" and "persons" were added as covariates in the analysis of tool concepts, none of the eight tracts remained significantly correlated (though a few still showed marginal significance). This means that those eight tracts are important but not unique to tool-processing, as they also contribute to the semantic processing of other object categories.

While the results and methodological approach used by Bi et al. (2015) are novel and valuable, it is worthwhile to consider a more detailed interpretation, with a specific focus on underlying semantic features of tool processing and their relation to the identified white matter tracts. The results of this study show that the eight tracts identified for tool concepts contribute to processing animals and persons too. It could be that these white matter tracts are linked to certain individual semantic features that are in- volved in tool processing but shared with other object categories, e.g., "form and surface" is a shared feature between tools and animals (McRae et al., 2005). Although the authors mention potential underlying features to some extent, these are not addressed in much detail. Nonetheless, their results contain information to consider semantic features' specificity and traits for certain white matter tracts.

With some of the involved gray matter regions linked to particular semantic features in previous research, we can make an educated guess as to what extent certain information might be related to the individual white matter connections. For example, the left pMTG is associated with the feature "biological motion," the left VPMC with "function," the left IPL with "contact," and the left DLPMC with features of "tactile" and "motor-action" (Cree and McRae, 2003; Kemmerer et al., 2008; Lin et al., 2011). The white matter tract between the left DLMPC and left VPMC might therefore carry information about how tactile and motor information can be functionally integrated. Indeed, in Bi et al. (2015), this tract's integrity (both lesion percentages and mean FA values) is significantly correlated with postural accuracy. Moreover, in their study, three out of the four white matter tracts that run to the left VPMC were marginally significant (left SPL-left VPMC regarding lesion percentages and left IPLleft VPMC and left pMTG-left VPMC regarding mean FA values) for tool concepts with "animals" and "persons" as covariates. A possible explanation could be that the feature "function" activates the left VPMC, which is considered to be a distinctive feature of "tools," but not of "animals" or "persons" (Cree and McRae, 2003). Lastly, as all action processing implicates the left IFG (Kemmerer et al., 2008) and the feature "contact" engages the left IPL, the left IFG-left IPL tract might carry information about how contacting an object is involved in an activity. Bi et al. (2015) indeed define this tract as having a central position in tool selectivity, but it may be more appropriate to highlight the specific involvement the distinctive feature "contact" for tool processing regarding this tract.

Another consideration is that the extent to which a semantic feature is involved in processing semantic knowledge is dependent on the specific task and context (Lebois et al., 2014). For example, when distinguishing which objects are hedge shears or a carpenter's pincer, visual features including shape and size might be more important than when one is asked to demonstrate their use, since roughly the same movement should be produced for using both tools independent of exact shape and size. Bi et al. (2015)_ using some tools with roughly similar actions (e.g., a brush and a broom both require stroking)-interpret their results as showing a dissociation between two networks, one for tool use and one for tool concept. However, an alternative interpretation is that there is one shared network in which features are represented with a variable strength on a scale from strong to weak, depending on the specific task. For example, knowledge of the feature "motor-action" associated with a brush and a broom might be weakly activated when naming them or judging their semantic similarities and differences, but strongly activated when demonstrating their use. This idea is supported by Bi et al.'s (2015) results, when the difference in partial correlations is calculated between the original value and the value once controlled for covariates (Table 1). The correlations of tool use with tract integrity (both lesion percentages and mean FA values) are only slightly affected by tool concepts as a covariate, while those of tool concept become substantially smaller with tool use as a covariate in the left DLPMC-left IFG and left DLPMC-left VPMC tracts, but not the left IFG-left IPL tract. This may indicate a stronger contribution of action-related features (e.g., "motor-action" associated with the left DLPMC) in tool actions than in tool objects.

Bi et al. (2015) highlight two main findings of their study, namely the anatomical tool-network and a notable division between the two networks that process tool use versus tool concepts. However, their data provide grounds for a more in-depth interpretation linking separate semantic features to distinct pathways, as well as the contribution of variable strengths of information depending on the context. In other words, the "tool" network might not be as tool-specific or divided between actions and objects as previously thought. Further 
research is needed to explore the mechanisms of white matter tracts in semantic processing, especially considering narrower semantic features and their characteristics through which humans build up conceptual knowledge.

\section{References}

Bi Y, Han Z, Zhong S, Ma Y, Gong G, Huang R, Song L, Fang Y, He Y, Caramazza A (2015)

The white matter structural network underlying human tool use and tool understanding. J Neurosci 35:6822-6835. CrossRef Medline

Cree GS, McRae K (2003) Analyzing the factors underlying the structure and computation of the meaning of chipmunk, cherry, chisel, cheese, and cello (and many other such con- crete nouns). J Exp Psychol Gen 132:163-201. CrossRef Medline

Kemmerer D, Castillo JG, Talavage T, Patterson S, Wiley C (2008) Neuroanatomical distribution of five semantic components of verbs: evidence from fMRI. Brain Lang 107:16-43. CrossRef Medline

Lebois LA, Wilson-Mendenhall CD, Barsalou LW (2014) Are automatic conceptual cores the gold standard of semantic processing? The context-dependence of spatial meaning in grounded congruency effects. Cogn Sci. Advance online publication. doi:10.1111/ cogs.12174. CrossRef Medline

Lewis JW (2006) Cortical networks related to human use of tools. Neuroscientist 12:211231. CrossRef Medline

Libon DJ, Rascovsky K, Powers J, Irwin DJ, Boller
A, Weinberg D, McMillan CT, Grossman M (2013) Comparative semantic profiles in semantic dementia and Alzheimer's disease. Brain 136:2497-2509. CrossRef Medline

Lin N, Lu X, Fang F, Han Z, BiY (2011) Is the semantic category effect in the lateral temporal cortex due to motion property differences? Neuroimage 55 : 1853-1864. CrossRef Medline

Mahon BZ, Caramazza A (2008) A critical look at the embodied cognition hypothesis and a new proposal for grounding conceptual content. J Physiol Paris 102:59-70. CrossRef Medline

McRae K, Cree GS, Seidenberg MS, McNorgan C (2005) Semantic feature production norms for a large set of living and nonliving things. Behav Res Methods 37:547-559. CrossRef Medline 